\title{
Contextual fibre growth to generate realistic axonal packing for diffusion MRI simulation
}

\author{
Ross Callaghan ${ }^{1}$, Daniel C. Alexander ${ }^{1}$, Hui Zhang ${ }^{1 \star}$, and Marco Palombo ${ }^{1 \star}$ \\ Centre for Medical Image Computing, Department of Computer Science, University \\ College London, London, UK \\ ross.callaghan.16@ucl.ac.uk
}

\begin{abstract}
This paper presents ConFiG, a method for generating white matter (WM) numerical phantoms with more realistic orientation dispersion and packing density. Numerical phantoms are commonly used in the validation of diffusion MRI (dMRI) techniques so it is important that they are as realistic as possible. Current numerical phantoms either oversimplify the complex morphology of WM or are unable to produce realistic orientation dispersion at high packing density. The highest packing density and orientation dispersion achieved so far is only $20 \%$ at $10^{\circ}$. ConFiG takes advantage of a shift of paradigm: rather than 'packing fibres', our algorithm 'grows fibres' contextually and efficiently, attempting to produce a substrate with desired morphological priors (orientation dispersion, packing density and diameter distribution), whilst avoiding intersection between fibres. The potential of ConFiG is demonstrated by reaching the highest packing density and orientation dispersion ever, to our knowledge $\left(25 \%\right.$ at $\left.35^{\circ}\right)$. The algorithm is compared with a 'brute force' growth approach showing that it is much more efficient, being $\mathcal{O}(n)$ compared to the $\mathcal{O}\left(n^{2}\right)$ brute-force method. The application of the method to dMRI is demonstrated with simulations of diffusion-weighted MR signal in three example substrates with differing orientation-dispersions, packing-densities and permeabilities.
\end{abstract}

\section{Introduction}

Numerical phantoms have found much use for validating many magnetic resonance imaging (MRI) experiments. In particular, many studies employing diffusion MRI (dMRI) to study microstructural features of white matter (WM) use numerical phantoms as part of the validation process $[16,9,10]$.

Typically, the models used in these studies represent axons in WM using simplistic geometrical representations such as parallel cylinders with uniform[9] or polydisperse [5] radii. Other studies introduce more complexity into the numerical phantoms with, for example, harmonic beading[1], spines [13] and undulation of individual fibres[11]. These studies typically only consider one mode of morphological variation at a time and all of these representations over-simplify the true

\footnotetext{
* Joint senior author
} 
complexity of axonal morphology that has been investigated through ex-vivo studies using electron microscopy[7].

Another emerging application of dMRI simulations is in the direct estimation of microstructural features from a measured dMRI signal. Some recent works use fingerprinting-style techniques and machine learning to match simulated signals and the corresponding ground truth microstructure of the numerical phantom to the measured signal[14, $6,12,8]$.

For all of these applications, synthetic models of WM that accurately represent real tissue are highly important.

Generating realistic WM numerical phantoms which accurately capture realistic microstructural features (such as dispersion, undulation, beading, etc.) at high packing densities is a major open challenge for the dMRI community. While densely packing straight, parallel, fibres is relatively easy, only a few groups have attempted to densely pack irregular, non-parallel, fibres. The highest packing density achieved so far under modest dispersion (up to $10^{\circ}$ ) reaches only $20 \%$ [4]. These approaches typically involve the packing of fibres. That is, trying to pack a set of existing fibres together as densely as possible.

Here, we propose a completely different strategy: rather than densely 'packing' irregular fibres, we 'grow' fibres contextually, mimicking natural fibre genesis. The algorithm presented proposes a method called ConFiG (Contextual Fibre Growth) for the generation of WM numerical phantoms with more realistic orientation dispersion and packing density. Fibres are grown one-by-one following a cost function which attempts to impose the morphological priors that are input to the algorithm.

The rest of the paper is organized as follows: Section 2 describes ConFiG, Section 3 details some experiments showing the potential of the algorithm and comparing it to a brute-force approach to fibre growth and Section 4 summarises the contributions and discusses future work.

\section{ConFiG: Contextual Fibre Growth}

In this section we describe ConFiG which grows fibres one-by-one avoiding intersection between fibres whilst attempting to ensure that the resulting substrate has desired morphological properties such as orientation dispersion, diameter distribution and packing density. The algorithm is broken into a few stages:

- the definition of inputs to the algorithm

- the generation of the network on which fibres grow

- the method by which each fibre grows

- the meshing procedure to create 3D meshes

Pseudocode for the first three of these points is shown in Algorithm 1. The rest of this section details each of the above stages. 


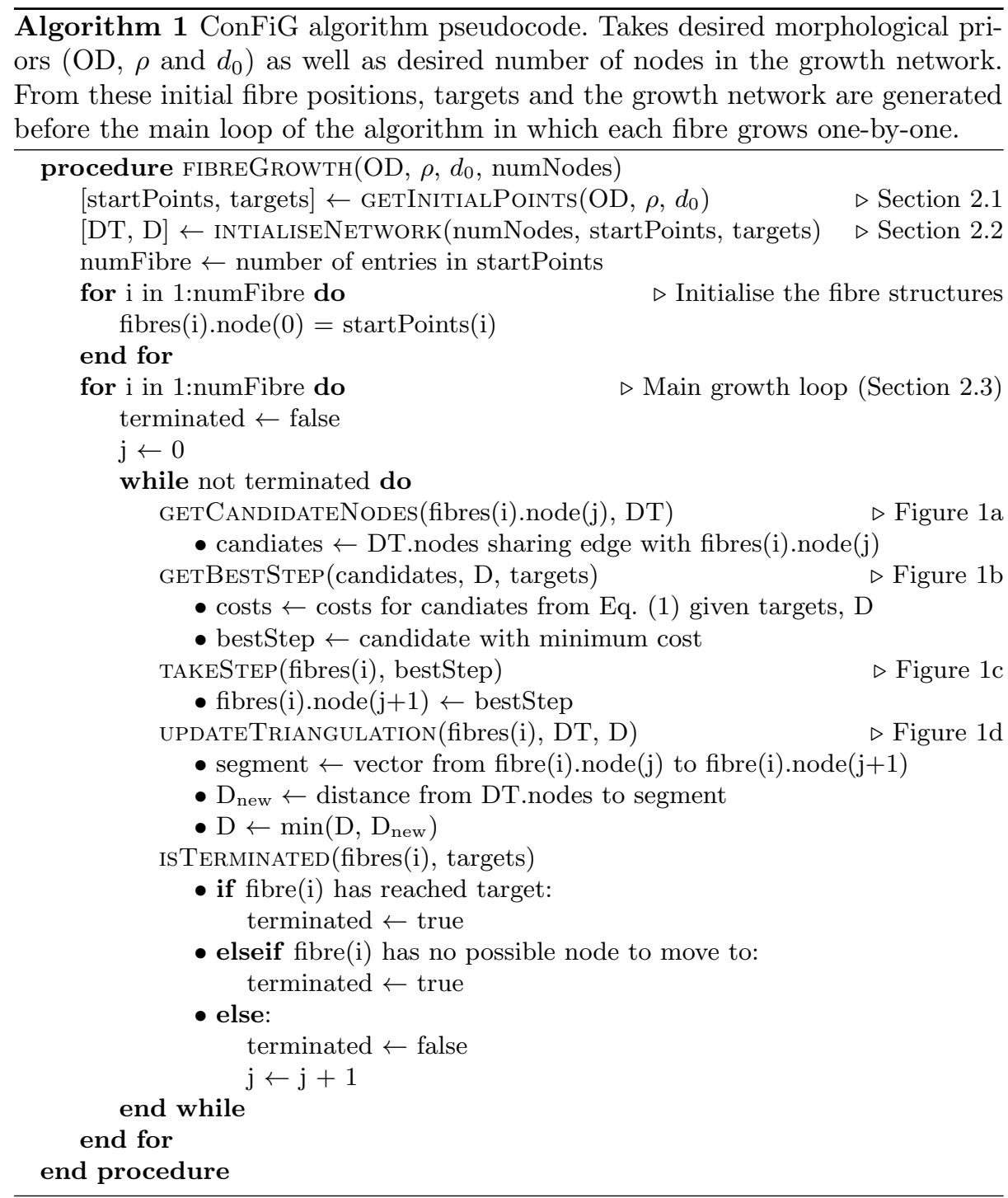

\subsection{Input to the algorithm}

The morphology of the final substrate will depend on the inputs to the algorithm which can be split into two general categories: parameters defining the fibre population(s), and parameters defining the space in which fibres grow.

Fibre parameters include the desired orientation dispersion (OD), packing density $(\rho)$ and diameter distribution $\left(P\left(d_{0}\right)\right)$. These three parameters determine the initial settings for each individual fibre. Each fibre is defined by a starting point and a target point towards which it will grow as well as an initial fibre diameter, $d_{0}$. These parameters for each fibre are determined from OD, $\rho$ and 
$P\left(d_{0}\right)$ by packing circles with the diameters drawn from $P\left(d_{0}\right)$ up to a density of $\rho$ in 2 dimensions. Orientation dispersion is introduced by moving the target points of fibres relative to the starting points.

Alternatively, if the user wishes, the starting point, target point and diameter for each fibre can be directly input, rather than allowing ConFiG to generate them, in order to specify particular fibre configurations such as crossing fibre bundles or fanning fibres.

Each fibre is allowed to shrink its diameter if it is necessary to fit into spaces close to other fibres. The maximum amount of shrinkage permitted is a controllable parameter, specified as a percentage of the initial fibre diameter.

Due to the stochastic nature of the algorithm, the final substrate is not guaranteed to have the exact morphological properties as input in the priors, however these inputs give the target morphology that ConFiG will attempt to produce.

Parameters defining the space in which the fibres grow are used to define a discretisation of the space into a set of node points that the fibres can occupy. Ideally, the space in which the fibres can grow is a continuous space, so there are an infinite number of positions a fibre can occupy, however this is impractical, so in this algorithm the space is discretised into a finite set of nodes.

Naturally, the choice of the density and arrangement of node points will impact the substrate that is produced. Too few nodes will result in fibres that have very long, straight segments and may introduce intersections between fibres. Using more nodes will reduce overlap between fibres at the cost of more memory usage and slower growth of the fibres. The arrangement of the nodes will also affect the morphology of the final substrate. For instance, placing nodes on a uniform grid may produce fibres with unnaturally angular paths. If the density of nodes on a uniform grid becomes sufficiently high, these angular bends are insignificant compared to the diameter and the fibres will have more natural shapes. For large substrates, the number of nodes required to satisfy this condition becomes intractably large. For this reason, the nodes used are typically pseudo-randomly distributed to ensure broadly uniform coverage of the space, whilst keeping the number of nodes required lower.

\subsection{Creation of the Growth Network}

In order to embed information about the local environment at each node, the first step of the algorithm is generating the paths that fibres can take between the nodes as well as defining a maximum diameter that can be sustained at each node to avoid intersection which will be denoted by $d_{i}$, for a node, $i$. These paths define a network along which the fibres may grow.

The paths between nodes are defined by the Delaunay triangulation[3] of the nodes which creates a sparse network in which any node can be reached from any other node. This triangulation creates edges between nearby nodes, encoding information about the local connectivity at each node. Nodes that 
become occupied by a fibre will be inaccessible to any future fibres, which is one way in which intersection is minimised between fibres.

The maximum diameter, $d_{i}$, at each node encodes information on the amount of space available at each node. Where $d_{i}$ is small, that node is close to an existing fibre, so any subsequent fibre passing through that node will have to shrink its diameter to $d_{i}$ in order to prevent intersections. Allowing fibres to contextually shrink their diameter allows fibres to occupy spaces which would otherwise be unavailable.

\subsection{Growth of a Fibre}

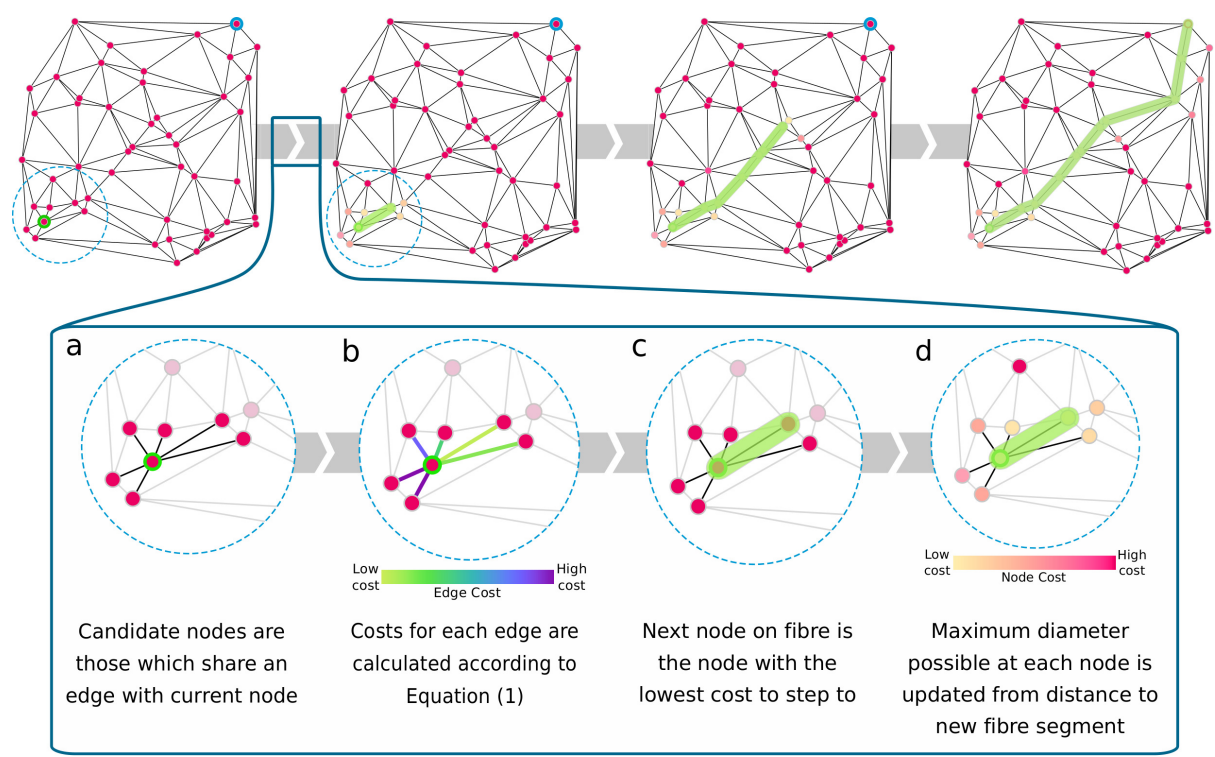

Fig. 1: Schematic overview of the fibre growth algorithm. A fibre grows sequentially, moving from one node to the next, starting from the start point (top left, green node) toward the target (top left, blue node) along the edges defined by the Delaunay triangulation. Inset: The algorithm determining which node a fibre steps to at any given iteration. a) The possible nodes to step to are those which share an edge with the current node. b) From the edges available costs are calculated using eqs. (1) and (2). c) The fibre will grow along the edge with the lowest cost. d) From this new segment, the maximum diameter sustainable at a given node is calculated, giving each node a cost based on the maximum sustainable diameter. This cost will then be used in the calculation of edge weights (b) for future fibres. Note that although this figure illustrates the algorithm in $2 \mathrm{D}$, in practice the algorithm grows fibres in $3 \mathrm{D}$.

Each individual fibre grows by moving the head of the fibre from node to node according to a cost function which attempts to ensure that the fibre moves 
towards its target whilst avoiding intersection. The main steps in the growth of a single fibre are shown in Figure 1.

The first step in the growth of a fibre is determining which nodes are the possible next nodes the fibre can step to, referred to as candidate nodes. From a given starting node, $s$, the candidate nodes are any of the nodes which share an edge with $s$.

The choice of which candidate node a fibre steps to from the current node is determined by a cost function. The cost function consists of two terms, one which penalises moving away from the target point, $t$, and one which penalises moving to a position where $d_{i}$ is low, meaning the fibre diameter would have to shrink. The cost function for a fibre at a position, $s$, to move to an candidate node, $c$, given a target point, $t$, is $l=l_{t}+f l_{d}$, where

$$
\begin{aligned}
& l_{t}=\frac{1}{2} \cdot \frac{\|s-c\|}{1+\|s-c\|} \cdot\left(1-\frac{(c-s) \cdot(t-s)}{\|c-s\|\|t-s\|}\right), \\
& l_{d}=\max \left(0, \frac{1}{d_{0}}\left(d_{0}-d_{i}\right)\right)
\end{aligned}
$$

$d_{0}$ is the desired radius of the fibre and $f$ is a weighting factor between the two terms. In this work, $f$ is fixed to 0.2 to more strongly weight growth toward the target.

Equation (1) is the term penalising moving away from the target. The dot product between the vector to the candidate and the vector to the target ensures that the minimum cost occurs when the candidate is directly aligned with the target. Equation (2) is the term penalising moving to a position where the radius of the fibre must shrink. For radii lower than the desired radius of the fibre, $d_{0}$, Equation (2) grows linearly with distance from $d_{0}$. For radii greater than or equal to $d_{0}$, Equation (2) is zero, meaning that regions of empty space are equally weighted.

The next node for a fibre will be the candidate node which has the lowest cost according to Equations (1) and (2). This method of finding a path through the triangulation by choosing the lowest cost node at each position amounts to a greedy best-first pathfinding approach with a heuristic given by Equations (1) and (2).

With the next node chosen, the value of $d_{i}$ needs to be updated for other nearby nodes. All nodes have $d_{i}$ set to the Euclidean distance between the node and the surface of the new section of fibre if that distance is less than the current value of $d_{i}$. This is illustrated in Figure 1d.

Any nodes which now lie within the fibre have $d_{i}$ set to zero. Nodes with $d_{i}=0$ are disallowed from future steps, meaning that once a fibre has grown, no future fibres can connect to any nodes within the fibre. This, in addition to shrinking the radius of future fibres according to $d_{i}$ at each node means that the fibres grow in an almost completely non-intersecting manner. Since the value of $d_{i}$ is set based on fibre-to-point distances, there can be cases in which the fibres would intersect when the closest point between two fibre sections is not at one of 
the fibre nodes. In order to account for this, a meshing process developed which can deform fibres around one another. This is described in Section 2.4.

The fibre growth algorithm will output a set of fibres which are defined by a series of nodes and the diameter of the fibre at each node. These are written into the Stockley-Wheal-Cole (SWC) format[15], a format commonly used to store cellular morphology information.

\subsection{Creation of Fibre Meshes}

In order to create 3D meshes to be used in dMRI simulations, a meshing process was developed using 3D modelling software Blender (https://blender.org). Fibres are meshed one-by-one using the Blender SWC Mesher add-on (https:// github.com/mcellteam/swc_mesher) which uses Blender metaballs to make a mesh.

In Blender, a metaball is an implicit surface defined as the isosurface of a so-called directing structure. This directing structure can be seen the source of a static field. For instance a spherical isosurface can be formed with a directing structure which mimics the electric field a point charge. When multiple metaballs come close to one another, the fields will combine to form a surface that merges the two spheres together. An example of metaball interactions is shown in Figure 2.

By placing metaballs along the skeleton of each fibre, with the path and diameters given from the fibre growth algorithm, a smooth surface is formed for each fibre. It is this implicit surface, created using metaballs that the SWC mesher add-on creates. This implicit surface can be turned into an explicit surface (i.e. a mesh of vertices and faces) in Blender, which can then be refined by progressively smoothing and reducing the number of faces in the mesh to create a mesh which can be used in dMRI simulations.

This process can be used to mesh each fibre individually, however issues can arise with intersection of fibres, as mentioned in Section 2.3. In order to account for this, a contextual meshing algorithm was developed. The metaball surface for one fibre is created using the SWC Mesher. This surface is then turned into a mesh as described above, however the metaballs are retained. The metaball potential is then turned negative, meaning that rather than attracting any future nearby metaball surfaces, it will repel them, as shown in Figure 2b. This means that subsequent fibres which are meshed very close to, or overlapping with existing fibres will deform organically to resolve the intersection, thus creating a series of completely non-intersecting fibre meshes which can be used by the dMRI simulator.

The deformation introduced by the contextual meshing process has two effects. As well as helping to prevent intersection between fibres, the deformation produces fibres with more organic non-circular cross sections, better mimicking realistic mythologies. This is vastly different to the majority of previous WM numerical phantoms which model fibres as circular or elliptic cylinders. 
a)
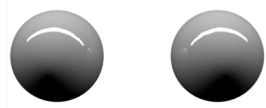

b)
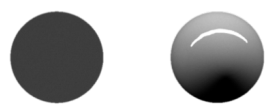

Separate (no interaction)
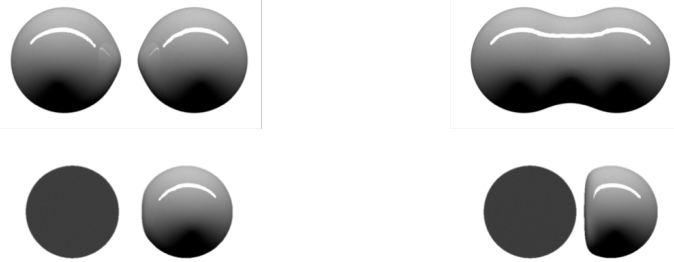

Close together (interaction)

Fig. 2: Simple example of metaball interactions. a) With two positive metaballs, the fields combine to attract the surfaces together. This is used to join individual segments into a continuous fibre. b) With one negative metaball (indicated by the flat grey circle) the surface of the metaball is repelled from the negative metaball. This is used to deform nearby fibres around one another.

\section{Experiments and Results}

\subsection{Demonstration of ConFiG}

To demonstrate the potential of ConFiG, three substrates at different (dispersion, packing density) conditions were generated: $\left(0^{\circ}, 60 \%\right),\left(15^{\circ}, 30 \%\right)$ and $\left(35^{\circ}, 25 \%\right)$, shown in Figure 3a. Each substrate is grown using $5 \times 10^{6}$ pseudorandomly placed source nodes for the growth network, giving a network with $3.88 \times 10^{7}$ edges and a mean distance between any given node and its neighbours of $0.29 \mu \mathrm{m}$. The packing densities chosen represent the highest densities achievable using ConFiG for each dispersion condition.

For the $0^{\circ}$ dispersed substrate, initial diameters were drawn from a gamma distribution with mean $d_{0}=2 \mu \mathrm{m}$ and standard deviation $\sigma_{d}=0.2 \mu \mathrm{m}$. The $15^{\circ}$ and $35^{\circ}$ substrates were generated with $d_{0}=1.2 \mu \mathrm{m}$ and $\sigma_{d}=0.2 \mu \mathrm{m}$ in order to show the flexibility of ConFiG to generate substrates with different diameter distributions as well as orientation dispersion and packing density. Diameters were limited to be permitted to shrink to $25 \%$ of the original fibre diameter in order to fit into space.

For each substrate, the Pulsed-Gradient-Spin-Echo (PGSE) signal was simulated in Camino[2] using $5 \times 10^{5}$ diffusing spins and $5 \times 10^{3}$ discrete time steps, uniformly distributed with bulk-diffusivity $\mathrm{D}_{0}=2 \mu \mathrm{m}^{2} / \mathrm{ms}$. To show the range of simulation possibilities available, three different membrane permeabilities $(\kappa=0$, $0.0025,0.0050 \mu \mathrm{m} / \mathrm{ms}$ ) were also imposed. The simulated PGSE measurement parameters were: $\delta / \Delta=1 / 40 \mathrm{~ms}$ and $50 \mathrm{~b}$-values from 0 to $9 \mathrm{~ms} / \mu \mathrm{m}^{2}$ along x-, $\mathrm{y}$ - and z-directions.

The corresponding direction-averaged simulated PGSE signals at different permeabilities are shown with $\mathrm{SNR}=\infty$ in Figure $3 \mathrm{~b}$ and SNR $=20$ in Figure 3c. The signal decays to a lower value as the dispersion increases and density decreases, as expected. 

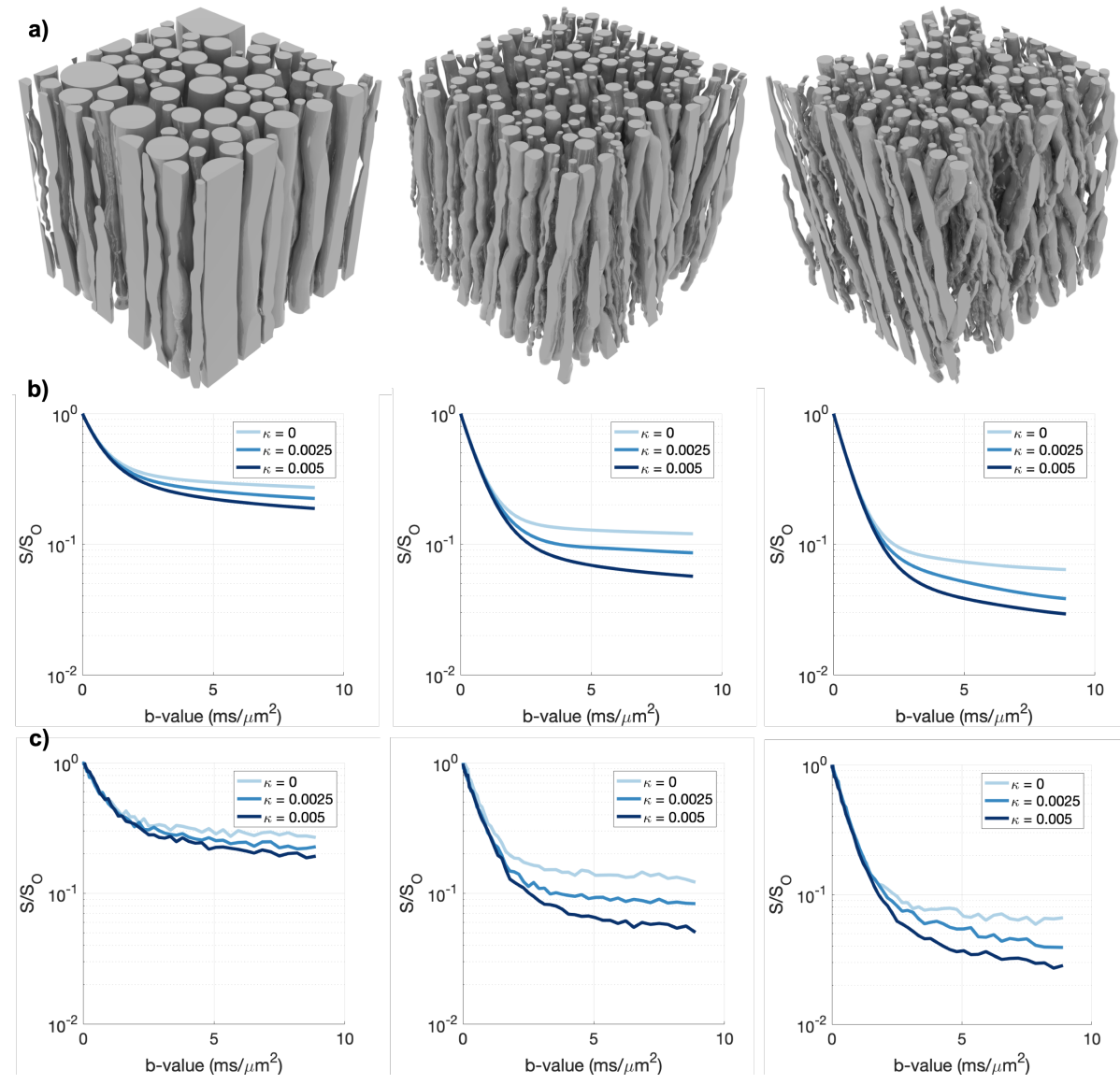

Fig. 3: a) Example substrates (cut into $30 \times 30 \times 30 \mu \mathrm{m}^{3}$ cube) from the fibre growth algorithm, left to right: Zero macroscopic dispersion (60\% density), $15^{\circ}$ of macroscopic dispersion ( $30 \%$ density), $35^{\circ}$ dispersed ( $25 \%$ density). b) Simulations for each substrate for varying permeabilities with $\mathrm{SNR}=\infty$ and c) SNR $=20$. Units of $\kappa$ are $\mu \mathrm{m} / \mathrm{ms}$.

\subsection{Comparison with Brute-Force Approach}

ConFiG was compared against the naïve brute-force approach to fibre growth. The brute-force approach grows fibres one segment at a time and checks for collisions between the new segment and all existing fibres. Each new segment is chosen from one of 128 candidate directions on a cone aligned with the previous segment, with each direction being weighted according to Equation (1).

Substrates were grown with both the brute-force approach and ConFiG using the same starting and target points and initial diameters. These initial parameters were determined by packing circles with gamma distributed radii (mean $d_{0}=2 \mu \mathrm{m}$, standard deviation $\sigma=0.6 \mu \mathrm{m}$ ) into a $40 \mu \mathrm{m} \times 40 \mu \mathrm{m}$ square up to a packing density of $60 \%$. Target points were set as $40 \mu \mathrm{m}$ directly above the 


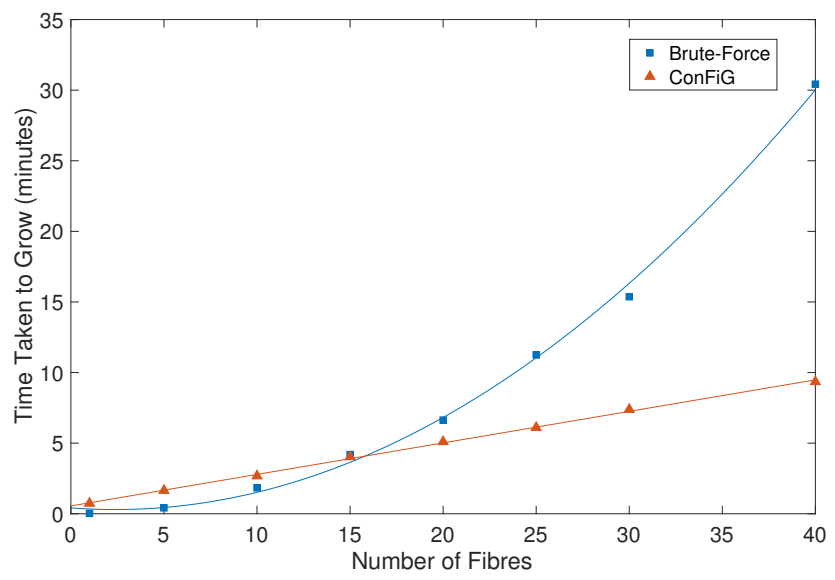

Fig. 4: Timing of brute force growth vs. the fibre growth algorithm along with a quadratic fit (brute-force) and linear fit (fibre growth algorithm). The fibre growth algorithm is clearly linear in the number of fibres, while brute force growth fits an order $n^{2}$ well.

starting points to define a substrate with $0^{\circ}$ macroscopic orientation dispersion. This resulted in a substrate with a total of 54 initial fibres.

The fibre growth algorithm used $1 \times 10^{6}$ randomly distributed points for the Delaunaty triangulation giving a mean distance between points of $0.5 \mu \mathrm{m}$, matching the brute force approach which used a segment length of $0.5 \mu \mathrm{m}$ for each new fibre segment.

From these initial parameters, fibres were grown using a subset of $n=$ $1,5,10,15,20,25,30,40$ fibres and the growth was timed. Each value of $n$ was timed 5 times with and the mean taken to reduce single-run timing fluctuations.

Figure 4 shows the timing results of the brute-force approach versus the fibre growth algorithm. The fibre growth algorithm has approximately $\mathcal{O}(n)$ complexity with $n$ being the number of fibres. Conversely, the brute-force algorithm shows $\mathcal{O}\left(n^{2}\right)$ complexity owing to the fact that every new segment has to check for collisions with all existing fibres.

The fibre growth algorithm has a higher $n=0$ offset which is caused by the overhead in calculating the Delaunay triangulation for the growth network. This causes the brute-force approach to have better performance at low $n$, while at higher $n$ (approaching the $>100$ fibres needed for a realistic dMRI voxel) the linearity of the fibre growth algorithm gives it much faster performance.

\section{Discussion and Conclusion}

ConFiG shifts perspective from previous works attempting to pack together fibres, by trying to mimic natural fibre genesis. This approach represents a major step towards very high fibre packing, enabling us to reach the highest dispersion 
at the highest packing density reached so far, to our knowledge. Our $\left(15^{\circ}, 30 \%\right)$ and $\left(35^{\circ}, 25 \%\right)$ represent an average $\sim 50 \%$ and $\sim 200 \%$ improvement, respectively, over the best previously reported results of $\left(10^{\circ}, 20 \%\right)[4]$.

The substrates presented in Figure 3 are just a few examples of the kinds of substrates that can be produced using our ConFiG method. By varying the setup of the morphological controls and start and target points, many different fibre configurations can be produced. Currently, fibres will attempt to grow in a straight line between the start and target points, meaning that certain configurations such as kissing bundles cannot be represented. However, the algorithm can in principle be extended to allow for series of target points, allowing the definition of a desired 'path' of a fibre.

Additionally, some input parameter settings cannot be achieved. For instance, trying to grow a substrate with both very high density and very high dispersion will result in a final substrate that does not reach the density required. The reason for this could be a combination of limitations of the algorithm in restricting growth to a discrete network and also the fact that some morphological settings are practically infeasible. This limitation, however, also applies to the fibre packing and brute force growth approaches.

One weakness of the fibre-growth algorithm is that since the fibre diameters are calculated from a fibre-to-point distance, there can still be some small amount of overlap between fibres. This is solved using the meshing process in Blender to deform the regions of slight overlap between neighbouring fibres.

To conclude, the proposed ConFiG approach, using the fully connected growth network, is shown to be more efficient than a 'brute-force' growth approach. The fact that ConFiG is linear with the number of fibres makes it far more efficient for high numbers of fibres. For instance, a realistic voxel will need hundreds or thousands of fibres which will become impractically slow for the 'brute-force' approach, whilst remaining manageable for our algorithm. This efficiency, along with the high density and orientation dispersion achieved means that ConFiG represents a promising step forward in the construction of ultra-realistic numerical phantoms of WM.

\section{Acknowledgements}

The he final authenticated version is available online at https://doi.org/10.1007/9783-030-20351-1_33.

This work is supported by the EPSRC-funded UCL Centre for Doctoral Training in Medical Imaging (EP/L016478/1) and the Department of Healths NIHR-funded Biomedical Research Centre at University College London Hospitals. This work was supported by EPSRC grants EP/M020533/1 and EP/N018702/1

\section{References}

1. Budde, M.D., Frank, J.A.: Neurite beading is sufficient to decrease the apparent diffusion coefficient after ischemic stroke. PNAS 107(32), 14472-14477 (2010). 
https://doi.org/10.1073/pnas.1004841107/

2. Cook, P.a., Bai, Y., Seunarine, K.K., Hall, M.G., Parker, G.J., Alexander, D.C.: Camino: Open-Source Diffusion-MRI Reconstruction and Processing. 14th Scientific Meeting of the International Society for Magnetic Resonance in Medicine 14, 2759 (2006)

3. Delaunay, P.B.: Sur la sphere vide. Bulletin of Academy of Sciences of the USSR (1934). https://doi.org/10.1051/jphysrad:01951001207073500

4. Ginsburger, K., Poupon, F., Beaujoin, J., Estournet, D., Matuschke, F., Mangin, J.F., Axer, M., Poupon, C.: Improving the realism of white matter numerical phantoms: A step toward a better understanding of the influence of structural disorders in diffusion MRI. Frontiers in Physics 5(FEB), 1-18 (2018). https://doi.org/10.3389/fphy.2018.00012

5. Hall, M.G., Alexander, D.C.: Convergence and Parameter Choice for Monte-Carlo Simulations of Diffusion MRI. IEEE Transactions on Medical Imaging 28(9), 13541364 (2009). https://doi.org/10.1109/TMI.2009.2015756

6. Hill, I., Palombo, M., Santin, M., Branzoli, F., Philippe, A.C., Wassermann, D., Aigrot, M.S., Stankoff, B., Zhang, H., Lehericy, S., Petiet, A., Alexander, D.C., Ciccarelli, O., Drobnjak, I.: Deep neural network based framework for in-vivo axonal permeability estimation. In: 26th Annual Meeting of the International Society for Magnetic Resonance in Medicine (ISMRM) (2018)

7. Lee, H.H., Yaros, K., Veraart, J., Pathan, J., Liang, F.X., Kim, S.G., Novikov, D.S., Fieremans, E.: Electron microscopy 3-dimensional segmentation and quantification of axonal dispersion and diameter distribution in mouse brain corpus callosum. bioRxiv 357491 (2018)

8. Nedjati-Gilani, G.L., Schneider, T., Hall, M.G., Cawley, N., Hill, I., Ciccarelli, O., Drobnjak, I., Wheeler-Kingshott, C.A., Alexander, D.C.: Machine learning based compartment models with permeability for white matter microstructure imaging. NeuroImage (2017). https://doi.org/10.1016/j.neuroimage.2017.02.013

9. Nilsson, M., Alerstam, E., Wirestam, R., Ståhlberg, F., Brockstedt, S., Lätt, J.: Evaluating the accuracy and precision of a two-compartment Karger model using Monte Carlo simulations. Journal of Magnetic Resonance 206(1), 59-67 (2010). https://doi.org/10.1016/j.jmr.2010.06.002

10. Nilsson, M., Lasič, S., Drobnjak, I., Topgaard, D., Westin, C.F.: Resolution limit of cylinder diameter estimation by diffusion MRI: The impact of gradient waveform and orientation dispersion. NMR in Biomedicine 30(7), 1-13 (2017). https://doi.org/10.1002/nbm.3711

11. Nilsson, M., Lätt, J., Ståhlberg, F., van Westen, D., Hagslätt, H.: The importance of axonal undulation in diffusion MR measurements: A Monte Carlo simulation study. NMR in Biomedicine 25(5), 795-805 (2012). https://doi.org/10.1002/nbm.1795

12. Palombo, M., Hill, I., Santin, M.D., Branzoli, F., Philippe, A.C., Wassermann, D., Aigrot, M.S., Stankoff, B., Zhang, H., Lehericy, S., Petiet, A., Alexander, D.C., Drobnjak, I.: Machine learning based estimation of axonal permeability: validation on cuprizone treated in-vivo mouse model of axonal demyelination. In: Proc. Joint Annual Meeting ISMRM-ESMRMB, Paris, France (2018)

13. Palombo, M., Ligneul, C., Hernandez-Garzon, E., Valette, J.: Can we detect the effect of spines and leaflets on the diffusion of brain intracellular metabolites? NeuroImage (2018). https://doi.org/10.1016/j.neuroimage.2017.05.003

14. Rensonnet, G., Scherrer, B., Girard, G., Jankovski, A., Warfield, S.K., Macq, B., Thiran, J.P., Taquet, M.: Towards microstructure fingerprinting: Estimation of tissue properties from a dictionary of Monte Carlo 
diffusion MRI simulations. NeuroImage 184(May 2018), 964-980 (2018). https://doi.org/10.1016/J.NEUROIMAGE.2018.09.076

15. Stockley, E., Cole, H., Brown, A., Wheal, H.: A system for quantitative morphological measurement and electronic modelling of neurons: three-dimensional reconstruction. J Neurosci Methods 47(1-2), 39-51 (1993)

16. Zhang, H., Schneider, T., Wheeler-Kingshott, C.A., Alexander, D.C.: NODDI: Practical in vivo neurite orientation dispersion and density imaging of the human brain. NeuroImage 61(4), 1000-1016 (2012). https://doi.org/10.1016/j.neuroimage.2012.03.072 